International Journal of Pure and Applied Mathematics

Volume 114 No. 1 2017, 133-142

ISSN: 1311-8080 (printed version); ISSN: 1314-3395 (on-line version)

url: http://www.ijpam.eu

doi: 10.12732/ijpam.v114i1.11

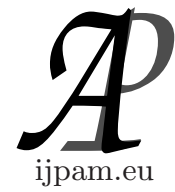

\title{
ON SEMI GENERALIZED STAR $b$-CONTINUOUS MAP IN TOPOLOGICAL SPACES
}

\author{
S. Sekar ${ }^{1 \S}$, B. Jothilakshmi ${ }^{2}$ \\ ${ }^{1}$ Department of Mathematics \\ Government Arts College (Autonomous) \\ Salem, 636 007, Tamil Nadu, INDIA \\ ${ }^{2}$ Department of Mathematics \\ Government Arts College (Autonomous) \\ Coimbatore, 641 045, Tamil Nadu, INDIA
}

\begin{abstract}
In this paper, we introduce a new class of semi generalized star $b$-continuous map and study some of their properties as well as inter relationship with other continuous maps.

AMS Subject Classification: 54C05, 54C08, 54C10

Key Words: $s g^{*} b$-continuous map, $b$-continuous map, $g b$ continuous map and $r g b$ continuous map
\end{abstract}

\section{Introduction}

Continuous map was studied for different types of closed sets by various researchers for past many years. In 1996, Andrijevic [3, 4] introduced new type called b-open sets. A.A. Omari and M.S.M. Noorani [2] were introduced and studied b-continuous map and b-closed map.

The aim of this paper is to continue the study of semi generalized star $b$ continuous map, semi generalized star $b$-closed map have been introduced and studied their relations with various generalized closed maps. Through out this

$\begin{array}{lr}\text { Received: } & \text { November 9, } 2016 \\ \text { Revised: } & \text { April 7, } 2017 \\ \text { Published: } & \text { April 21, 2017 }\end{array}$

(C) 2017 Academic Publications, Ltd. url: www.acadpubl.eu

$\S_{\text {Correspondence author }}$ 
paper $(X, \tau)$ and $(Y, \sigma)$ represent the non-empty topological spaces on which no separation axioms are assumed, unless otherwise mentioned.

Let $A \subseteq X$, the closure of $A$ and interior of $A$ will be denoted by $\operatorname{cl}(A)$ and $\operatorname{int}(A)$ respectively, union of all $b$ - open sets $X$ contained in $A$ is called $b$ interior of $A$ and it is denoted by $\operatorname{bint}(A)$, the intersection of all $b$ - closed sets of $X$ containing $A$ is called $b$ - closure of $A$ and it is denoted by $b c l(A)$.

\section{Preliminaries}

Definition 2.1. Let a subset $A$ of a topological space $(X, \tau)$, is called

1) a pre-open set [16] if $A \subseteq \operatorname{int}(\operatorname{cl}(A))$.

2) a semi-open set [13] if $A \subseteq \operatorname{cl}(\operatorname{int}(A))$.

3) a $\alpha$-open set [17] if $A \subseteq \operatorname{int}(\operatorname{cl}(\operatorname{int}(A)))$.

4) a $\alpha$ generalized closed set (briefly $\alpha g$ - closed) [14] if $\alpha c l(A) \subseteq U$ whenever $A \subseteq U$ and $U$ is open in $X$.

5) a generalized $*$ closed set (briefly $g^{*}$-closed) [20] if $\operatorname{cl}(A) \subseteq U$ whenever $A \subseteq U$ and $U$ is $\hat{g}$ open in $X$.

6) a generalized $b$ - closed set (briefly $g b$ - closed) [2] if $b c l(A) \subseteq U$ whenever $A \subseteq U$ and $U$ is open in $X$.

7) a generalized semi-pre closed set (briefly $g s p-\operatorname{closed})[9]$ if $\operatorname{spcl}(A) \subseteq U$ whenever $A \subseteq U$ and $U$ is open in $X$.

8) a generalized pre- closed set (briefly $g p$ - closed) [10] if $p c l(A) \subseteq U$ whenever $A \subseteq U$ and $U$ is open in $X$.

9) a generalized semi- closed set (briefly $g s$ - closed) [9] if $\operatorname{scl}(A) \subseteq U$ whenever $A \subseteq U$ and $U$ is open in $X$.

10) a semi generalized closed set (briefly $s g$ - closed) [6] if $\operatorname{scl}(A) \subseteq U$ whenever $A \subseteq U$ and $U$ is semi open in $X$.

11) a generalized pre regular closed set (briefly gpr-closed) [10] if $\operatorname{pcl}(A) \subseteq U$ whenever $A \subseteq U$ and $U$ is regular open in $X$.

12) a semi generalized $b$ - closed set (briefly $s g b$ - closed) [11] if $b c l(A) \subseteq U$ whenever $A \subseteq U$ and $U$ is semi open in $X$. 
13) a $\ddot{g}$ - closed set [19] if $\operatorname{cl}(A) \subseteq U$ whenever $A \subseteq U$ and $U$ is sg open in $X$.

14) a semi generalized star $b$ - closed set (briefly $s g^{*} b$ - closed) [20] if $b c l(A) \subseteq U$ whenever $A \subseteq U$ and $U$ is semi open in $X$.

Definition 2.2. A function $f:(X, \tau) \rightarrow(Y, \sigma)$, is called

1) a pre continuous map [16] if $f^{-1}(V)$ is pre open in $(X, \tau)$ for every open set $\mathrm{V}$ of $(Y, \sigma)$.

2) a semi continuous map [13] if $f^{-1}(V)$ is semi open in $(X, \tau)$ for every open set $\mathrm{V}$ of $(Y, \sigma)$.

3) a continuous map [17] if $f^{-1}(V)$ is open in $(X, \tau)$ for every open set $\mathrm{V}$ of $(Y, \sigma)$.

4) a generalized continuous map [14] if $f^{-1}(V)$ is generalized-closed in $(X, \tau)$ for every open set $\mathrm{V}$ of $(Y, \sigma)$.

5) a generalized b continuous map [2] if $f^{-1}(V)$ is $g b$-open in $(X, \tau)$ for every open set $\mathrm{V}$ of $(Y, \sigma)$.

6) a generalized semi-pre continuous map [9] if $f^{-1}(V)$ is $g s p$-open in $(X, \tau)$ for every open set $\mathrm{V}$ of $(Y, \sigma)$.

7) a semi generalized continuous map [6] if $f^{-1}(V)$ is $s g$-open in $(X, \tau)$ for every open set $\mathrm{V}$ of $(Y, \sigma)$.

8) a generalized pre regular continuous map [10] if $f^{-1}(V)$ is gpr-open in $(X, \tau)$ for every open set $\mathrm{V}$ of $(Y, \sigma)$.

9) a semi generalized b continuous map [11] if $f^{-1}(V)$ is $s g b$-open in $(X, \tau)$ for every open set $\mathrm{V}$ of $(Y, \sigma)$.

10) a regular generalized b continuous map [22] if $f^{-1}(V)$ is $r g b$-open in $(X, \tau)$ for every open set $\mathrm{V}$ of $(Y, \sigma)$.

\section{On Semi Generalized $s g^{*} b$-Continuous Map}

In this section, we introduce semi generalized star $b$-continuous map $\left(s g^{*} b\right.$ continuous) in topological spaces by using the notions of $s g^{*} b$-closed maps and study some of their properties. 
Definition 3.1. Let $X$ and $Y$ be two topological spaces. A map $f$ : $(X, \tau) \rightarrow(Y, \sigma)$ is called semi generalized star $b$-continuous map (briefly, $s g^{*} b$ continuous map) if the image of every closed set in $Y$ is $s g^{*} b$-open in $X$.

Theorem 3.2. If a map $f:(X, \tau) \rightarrow(Y, \sigma)$ from a topological space $X$ into a topological space $Y$ is continuous, then it is $s g^{*} b$-continuous.

Proof. Let $V$ be an open set in $Y$. Since $f$ is continuous, then $f^{-1}(V)$ is open in $X$. As every open set is $s g^{*} b$-open, $f^{-1}(V)$ is $s g^{*} b$-open in $X$. Therefore $f$ is $s g^{*} b$-continuous.

The converse of above theorem need not be true as seen from the following example.

Example 3.3. Consider $X=Y=\{a, b, c\}, \tau=\{X, \phi,\{b\},\{a, b\}\}$ and $\sigma=\{Y, \phi,\{a\},\{a, c\}\}$. Let $f:(X, \tau) \rightarrow(Y, \sigma)$ be defined by $f(a)=a, f(b)=$ $b, f(c)=c$, then $f$ is $s g^{*} b$-continuous but not continuously as the inverse image of an open set $\{a, c\}$ in $Y$ is $\{b, c\}$ which is not open set in $X$.

Theorem 3.4. Let $X$ and $Y$ be topological spaces. If a map $f:(X, \tau) \rightarrow$ $(Y, \sigma)$ is $\ddot{g}$-continuous, then it is $s g^{*} b$-continuous.

Proof. Let us assume that $f:(X, \tau) \rightarrow(Y, \sigma)$ is $b$-continuous. Let $V$ be an open set in $Y$, Since $f$ is $b$-continuous then $f^{-1}(V)$ is $b$-open. Hence every $b$-open is $s g^{*} b$-open in $X$. Therefore $f$ is $s g^{*} b$-continuous.

The converse of above theorem need not be true as seen from the following example.

Example 3.5. Consider $X=Y=\{a, b, c\}, \tau=\{X, \phi,\{a, b\}\}$ and $\sigma=$ $\{Y, \phi,\{a, c\}\}$. Let $f:(X, \tau) \rightarrow(Y, \sigma)$ be defined by $f(a)=c, f(b)=a, f(c)=b$. the $f$ is $s g^{*} b$-continuous but not $\ddot{g}$-continuous as the inverse image of an open set $\{a, c\}$ in $Y$ is $\{a, b\}$ which is not $\ddot{g}$-open set in $X$.

Theorem 3.6. Let $X$ and $Y$ be topological spaces. If a map $f:(X, \tau) \rightarrow$ $(Y, \sigma)$ is $\alpha$-continuous then it is $s g^{*} b$-continuous.

Proof. Let us assume that $f:(X, \tau) \rightarrow(Y, \sigma)$ is $\alpha$-continuous. Let $V$ be an open set in $Y$, Since $f$ is $\alpha$-continuous then $f^{-1}(V)$ is $\alpha$-open. Hence every $\alpha$-open is $s g^{*} b$-open in $X$. Therefore $f$ is $s g^{*} b$-continuous.

The converse of above theorem need not be true as seen from the following example. 
Example 3.7. Consider $X=Y=\{a, b, c\}, \tau=\{X, \phi,\{a, b\}\}$ and $\sigma=$ $\{Y, \phi,\{b\},\{a, b\}\}$. Let $f:(X, \tau) \rightarrow(Y, \sigma)$ be defined by $f(a)=a, f(b)=$ $c, f(c)=b$, then $f$ is $s g^{*} b$-continuous but not $\alpha$-continuously as the inverse image of an open set $\{a, b\}$ in $Y$ is $\{b, c\}$ which is not $\alpha$-open set in $X$.

Theorem 3.8. Let $X$ and $Y$ be topological spaces. If a map $f:(X, \tau) \rightarrow$ $(Y, \sigma)$ is semi continuous, then it is $s g^{*} b$-continuous.

Proof. Let us assume that $f:(X, \tau) \rightarrow(Y, \sigma)$ is semi continuous. Let $V$ be an open set in $Y$, Since $f$ is semi continuous then $f^{-1}(V)$ is semi open. Hence every semi open is $s g^{*} b$-open in $X$. Therefore $f$ is $s g^{*} b$-continuous.

The converse of above theorem need not be true as seen from the following example.

Example 3.9. Consider $X=Y=\{a, b, c\}, \tau=\{X, \phi,\{b\},\{a, b\}\}$ and $\sigma=\{Y, \phi,\{b\},\{b, c\}\}$. Let $f:(X, \tau) \rightarrow(Y, \sigma)$ be defined by $f(a)=c, f(b)=$ $a, f(c)=b$, then $f$ is $s g^{*} b$-continuous but not semi-continuously as the inverse image of an open set $\{b\}$ in $Y$ is $\{c\}$ which is not semi-open set in $X$.

Theorem 3.10. Let $X$ and $Y$ be topological spaces. If a map $f:(X, \tau) \rightarrow$ $(Y, \sigma)$ is pre continuous, then it is $s g^{*} b$-continuous.

Proof. Let us assume that $f:(X, \tau) \rightarrow(Y, \sigma)$ is pre continuous. Let $V$ be an open set in $Y$, Since $f$ is pre continuous, then $f^{-1}(V)$ is pre open. Hence every pre open is $s g^{*} b$-open in $X$. Therefore $f$ is $s g^{*} b$-continuous.

The converse of above theorem need not be true as seen from the following example.

Example 3.11. Consider $X=Y=\{a, b, c\}, \tau=\{X, \phi,\{b\},\{a, b\}\}$ and $\sigma=\{Y, \phi,\{a\},\{a, b\}\}$. Let $f:(X, \tau) \rightarrow(Y, \sigma)$ be defined by $f(a)=c, f(b)=$ $b, f(c)=a$, then $f$ is $s g^{*} b$-continuous but not pre-continuous as the inverse image of an open set $\{a\}$ in $Y$ is $\{c\}$ which is not pre-open set in $X$.

Theorem 3.12. Let $X$ and $Y$ be topological spaces. If a map $f:(X, \tau) \rightarrow$ $(Y, \sigma)$ is $\alpha g$ continuous, then it is $s g^{*} b$-continuous.

Proof. Let us assume that $f:(X, \tau) \rightarrow(Y, \sigma)$ is $\alpha g$ continuous. Let $V$ be an open set in $Y$, Since $f$ is $\alpha g$ continuous then $f^{-1}(V)$ is $g \alpha$-open. Hence every $g \alpha$-open is $s g^{*} b$-open in $X$. Therefore $f$ is $s g^{*} b$-continuous.

The converse of above theorem need not be true as seen from the following example. 
Example 3.13. Consider $X=Y=\{a, b, c\}, \tau=\{X, \phi,\{a, b\}\}$ and $\sigma=$ $\{Y, \phi,\{a\},\{a, c\}\}$. Let $f:(X, \tau) \rightarrow(Y, \sigma)$ be defined by $f(a)=b, f(b)=$ $a, f(c)=c$, then $f$ is $s g^{*} b$-continuous but not $\alpha g$-continuous as the inverse image of an open set $\{a, c\}$ in $Y$ is $\{b, c\}$ which is not $\alpha g$-open set in $X$.

Theorem 3.14. Let $X$ and $Y$ be topological spaces. If a map $f:(X, \tau) \rightarrow$ $(Y, \sigma)$ is $s g^{*} b$ continuous then it is gsp-continuous.

Proof. Let us assume that $f:(X, \tau) \rightarrow(Y, \sigma)$ is $s g^{*} b$ continuous. Let $V$ be an open set in $Y$, Since $f$ is $s g^{*} b$ continuous then $f^{-1}(V)$ is $s g^{*} b$ open. Hence every $s g^{*} b$ open is $g s p$ open in $X$. Therefore $f$ is $g s p$ continuous.

The converse of above theorem need not be true as seen from the following example.

Example 3.15. Let $X=Y=\{a, b, c\}, \tau=\{X, \phi,\{b\},\{b, c\}\}$ and $\sigma=$ $\{Y, \phi,\{a\},\{a, b\}\}$. Let $f:(X, \tau) \rightarrow(Y, \sigma)$ be defined by $f(a)=b, f(b)=$ $c, f(c)=a$, then $f$ is gsp continuous but not $s g^{*} b$ continuous as the inverse image of an open set $\{a\}$ in $Y$ is $\{c\}$ which is not $s g^{*} b$ open set in $X$.

Theorem 3.16. Let $X$ and $Y$ be topological spaces. If a map $f:(X, \tau) \rightarrow$ $(Y, \sigma)$ is $s g^{*} b$-continuous, then it is $g b$-continuous.

Proof. Let us assume that $f:(X, \tau) \rightarrow(Y, \sigma)$ is $s g^{*} b$-continuous. Let $V$ be an open set in $Y$, since $f$ is $s g^{*} b$-continuous then $f^{-1}(V)$ is $s g^{*} b$-open. Hence every $s g^{*} b$-open is $g b$-open in $X$. Therefore $f$ is $g b$-continuous.

The converse of above theorem need not be true as seen from the following example.

Example 3.17. Let $X=Y=\{a, b, c\}, \tau=\{X, \phi,\{c\},\{b, c\}\}$ and $\sigma=$ $\{Y, \phi,\{b\},\{a, c\}\}$. Let $f:(X, \tau) \rightarrow(Y, \sigma)$ be defined by $f(a)=a, f(b)=$ $b, f(c)=c$, then $f$ is $g b$-continuous but not $s g^{*} b$-continuous as the inverse image of an open set $\{b\}$ in $Y$ is $\{b\}$ is not $s g^{*} b$-open set in $X$.

Theorem 3.18. Let $X$ and $Y$ be topological spaces. If a map $f:(X, \tau) \rightarrow$ $(Y, \sigma)$ is $s g$ continuous, then it is $s g^{*} b$-continuous.

Proof. Let us assume that $f:(X, \tau) \rightarrow(Y, \sigma)$ is $s g$ continuous. Let $V$ be an open set in $Y$, Since $f$ is $s g$ continuous then $f^{-1}(V)$ is $s g$ open. Hence every $s g$ open is $s g^{*} b$ open in $X$. Therefore $f$ is $s g^{*} b$-continuous.

The converse of above theorem need not be true as seen from the following example. 
Example 3.19. Let $X=Y=\{a, b, c\}, \tau=\{X, \phi,\{a\},\{c\},\{a, c\}\}$ and $\sigma=\{Y, \phi,\{c\},\{b, c\}\}$. Let $f:(X, \tau) \rightarrow(Y, \sigma)$ be defined by $f(a)=c, f(b)=$ $a, f(c)=b$, then $f$ is $s g^{*} b$-continuous but not sg-continuous as the inverse image of an open set $\{b, c\}$ in $Y$ is $\{a, b\}$ which is not sg-open set in $X$.

Remark 3.20. The following examples show that $s g^{*} b$ - continuous and $g p$-continuous maps are independent.

Example 3.21. Let $X=Y=\{a, b, c\}, \tau=\{X, \phi,\{b\},\{c\},\{b, c\}\}$ and $\sigma=\{Y, \phi,\{a\},\{a, c\}\}$. Let $f:(X, \tau) \rightarrow(Y, \sigma)$ be defined by $f(a)=c, f(b)=$ $a, f(c)=b$, then $f$ is $s g^{*} b$-continuous but not $g p$-continuous as the inverse image of an open set $\{a, c\}$ in $Y$ is not gp-open set in $X$.

Example 3.22. Let $X=Y=\{a, b, c\}, \tau=\{X, \phi,\{c\}\}$ and $\sigma=\{Y, \phi,\{c\}$, $\{a, c\},\{b, c\}\}$. Let $f:(X, \tau) \rightarrow(Y, \sigma)$ be defined by $f(a)=a, f(b)=c, f(c)=b$, then $f$ is gp-continuous but not $s g^{*} b$-continuous as the inverse image of $\{c\}$ in $Y$ is $\{b\}$ which is not $s g^{*} b$-open set in $X$.

Remark 3.23. The following examples show that $s g^{*} b$ - continuous and gpr-continuous maps are independent.

Example 3.24. Let $X=Y=\{a, b, c\}, \tau=\{X, \phi,\{b\},\{a, b\},\{b, c\}\}$ and $\sigma=\{Y, \phi,\{a\},\{a, b\}\}$. Let $f:(X, \tau) \rightarrow(Y, \sigma)$ be defined by $f(a)=b, f(b)=$ $c, f(c)=a$, then $f$ is gpr-continuous but not $s g^{*} b$-continuous as the inverse image of $\{a, b\}$ in $Y$ is $\{a, c\}$ not $s g^{*} b$-open set in $X$.

Example 3.25. Let $X=Y=\{a, b, c\}, \tau=\{X, \phi,\{a\},\{b\},\{a, b\}\}$ and $\sigma=\{Y, \phi,\{b\},\{b, c\}\}$. Let $f:(X, \tau) \rightarrow(Y, \sigma)$ be defined by $f(a)=b, f(b)=$ $a, f(c)=c$, then $f$ is $s g^{*} b$-continuous but not gpr-continuous as the inverse image of $\{b, c\}$ in $Y$ is $\{a, c\}$ which is not gpr-open set in $X$.

Remark 3.26. The following examples show that $s g^{*} b$ - continuous and rgb-continuous maps are independent.

Example 3.27. Let $X=Y=\{a, b, c\}, \tau=\{X, \phi,\{a\},\{a, b\},\{b, c\}\}$ and $\sigma=\{Y, \phi,\{a, c\}\}$. Let $f:(X, \tau) \rightarrow(Y, \sigma)$ be defined by $f(a)=b, f(b)=$ $a, f(c)=c$, then $f$ is rgb-continuous but not $s g^{*} b$-continuous as the inverse image of $\{b, c\}$ in $Y$ is not $s g^{*} b$-open set in $X$.

Example 3.28. Let $X=Y=\{a, b, c\}, \tau=\{X, \phi,\{b\},\{c\},\{b, c\}\}$ and $\sigma=\{Y, \phi,\{a\},\{a, b\}\}$. Let $f:(X, \tau) \rightarrow(Y, \sigma)$ be defined by $f(a)=b, f(b)=$ $c, f(c)=a$, then $f$ is $s g^{*} b$-continuous but not $r g b$-continuous as the inverse image of $\{a, b\}$ in $Y$ is $\{a, c\}$ which is not rgb-open set in $X$.

Remark 3.29. The following examples show that $s g b$ - continuous and 
$s g^{*} b$-continuous maps are independent.

Example 3.30. Let $X=Y=\{a, b, c\}, \tau=\{X, \phi,\{a\},\{c\},\{a, c\},\{b, c\}\}$ and $\sigma=\{Y, \phi,\{a, b\}\}$. Let $f:(X, \tau) \rightarrow(Y, \sigma)$ be defined by $f(a)=a, f(b)=$ $c, f(c)=b$, then $f$ is $s g^{*} b$-continuous but not sgb-continuous as the inverse image of $\{a, b\}$ in $Y$ is not sgb-open set in $X$.

Example 3.31. Let $X=Y=\{a, b, c\}, \tau=\{X, \phi,\{a\},\{b\},\{a, b\},\{b, c\}\}$ and $\sigma=\{Y, \phi,\{a\},\{a, b\}\}$. Let $f:(X, \tau) \rightarrow(Y, \sigma)$ be defined by $f(a)=$ $b, f(b)=a, f(c)=c$, then $f$ is $s g b$-continuous but not $s g^{*} b$-continuous as the inverse image of $\{a, b\}$ in $Y$ is $\{a, c\}$ which is not $s g^{*} b$-open set in $X$.

\section{Applications}

Theorem 4.1. If a map $f:(X, \tau) \rightarrow(Y, \sigma)$ then

(i) the following are equivalent

(a) $f$ is $s g^{*} b$ - continuous

(b) The inverse image of open set in $Y$ is $s g^{*} b$ - open in $X$.

(ii) If $f:(X, \tau) \rightarrow(Y, \sigma)$ is $s g^{*} b$ - continuous, then $f\left(b^{*}(A)\right) \subset \operatorname{cl}(f(A))$ for every subset $A$ of $X$

Proof. (i) Let us assume that $f: X \rightarrow Y$ be $s g^{*} b$ - continuous. Let $F$ be open in $Y$. Then $F^{c}$ is closed in $Y$. Since $f$ is $s g^{*} b$ - continuous, $f^{-1}\left(F^{c}\right)$ is $s g^{*} b$ - closed in $X$. But $f^{-1}\left(F^{c}\right)=X-f^{-1}(F)$. Thus $X-f^{-1}(F)$ is $s g^{*} b$ closed in $X$. So $f^{-1}(F)$ is $s g^{*} b$ - open in $X$. Hence (a) $\Rightarrow(\mathrm{b})$.

Conversely, let us assume that the inverse image of each open set in $Y$ is $s g^{*} b$ open in $X$. Let $G$ be closed in $Y$. Then $G^{c}$ is open in $Y$. By assumption $X-f^{-1}(G)$ is open in $X$. So $f^{-1}(G)$ is $s g^{*} b$ - closed in $X$. Therefore $f$ is $s g^{*} b$ - continuous. Hence (b) $\Rightarrow(\mathrm{a})$. We have (a) and (b) are equivalent.

(ii) Let us assume that $f$ is $s g^{*} b$ - continuous. Let $A$ be any subset of $X$. Then $c l(f(A))$ is closed in $Y$. Since $f$ is $s g^{*} b$ - continuous, $f^{-1}(c l(f(A)))$ is $s g^{*} b$ - closed in $X$ and it contains $A$. But $b^{*}(A)$ is the intersection of all $b^{*}$ closed sets containing. Therefore $b^{*}(A) \subset f^{-1}(c l(f(A)))$. So that $f\left(b^{*}(A)\right) \subset c l(f(A))$.

Theorem 4.2 (PASTING LEMMA for $s g^{*} b$-continuous maps). Let $X=$ $A \cup B$ be a topological space with topology $\tau$ and $Y$ be a topological space with topology $\sigma$. Let $f:(A, \tau / A) \rightarrow(Y, \sigma)$ and $g:(B, \tau / B) \rightarrow(Y, \sigma)$ be $s g^{*} b$ - continuous map such that $f(x)=g(x)$ for every $x \in A \cup B$. Suppose that $A$ and $B$ are $s g^{*} b$ - closed sets in $X$, then $\alpha:(X, \tau) \rightarrow(Y, \sigma)$ is $s g^{*} b$ - continuous. 
Proof. Let $F$ be any closed set in $Y$. Clearly $\alpha^{-1}(F)=f^{-1}(F) \cup g^{-1}(F)=$ $C \cup D$, where $C=f^{-1}(F)$ and $D=g^{-1}(F)$. But $C$ is $s g^{*} b$ - closed in $A$ and $A$ is $s g^{*} b$ - closed in $X$. So $C$ is $s g^{*} b$ - closed in $X$. Since we have prove the result, if $B \subseteq A \subseteq X, B$ is $s g^{*} b$ - closed in $A$ and $A$ is $s g^{*} b$ - closed in $X$, then $B$ is $s g^{*} b$ - closed in $X$. Also $C \cup D$ is $s g^{*} b$ - closed in $X$. Therefore $\alpha^{-1}(F)$ is $s g^{*} b$ - closed in $X$. Hence $\alpha$ is $s g^{*} b$ - continuous.

\section{References}

[1] M.E. Abd El-Monsef, S.N. Deeb and R.A. Mahmoud, $\beta$-open sets and $\beta$-continous mappings, Bull. Fac. Sci. Assiut. Univ., 12 (1983), 77-90.

[2] Ahmad Al-Omari and Mohd. Salmi Md. Noorani, On generalized b-closed sets, Bull. Malays. Math. Sci. Soc. (2), 32, No. 1 (2009), 19-30.

[3] D. Andrijevic, Semi-pre open sets, Mat. Vesnik, 38, No. 1 (1986), 24-32.

[4] D. Andrijevic, b-open sets, Mat. Vesink, 48 (1996), 59-64.

[5] S.P. Arya and T. Nour, Characterizations of $s$-normal spaces, Indian J. Pure Applied Maths., 21, No. 8 (1990), 717-719.

[6] P. Bhattacharya and B.K. Lahiri, Semi-generalized closed sets on topology, Indian J. Maths., 29, No. 3 (1987), 375-382.

[7] M. Caldas and S. Jafari, On some applications of b-open sets in topological spaces, Kochi J. Math., 24, No. 4 (1998), 681-688.

[8] G. Dimaio and T. Noiri, On s-closed spaces, Indian J. Pure Appl. Math., 18, No. 3 (1987), 226-233.

[9] J. Dontchev, On generalized semi-pre open sets, Mem. Fac. Sci. Kochi. Univ., Ser. A. Math., 16 (1995), 35.

[10] Y. Gnanambal, On generalized pre-regular closed sets in topological spaces, Indian J. Pure Appl. Math., 28 (1997), 351-360.

[11] D. Iyappan and N. Nagaveni, On semi generalized b-closed set, Nat. Sem. on Math. and Comp. Sci., (2010), Proc. 6.

[12] N. Levine, Generalized closed sets in topology, Tend Circ., Mat. Palermo (2), 19 (1970), 89-96.

[13] N. Levine, Semi-open sets and semi-continuity in topological spaces, Amer. Math. Monthly, 70 (1963), 36-41.

[14] H. Maki, R. Devi and K. Balachandran, Associated topologies of generalized $\alpha$-closed sets and $\alpha$-generalized closed sets, Mem. Fac. Sci. Kochi. Univ. Ser. A. Math., 15 (1994), 51-63.

[15] H. Maki, R.J. Umehara and T. Noiri, Every topological space is pre-T 1/2, Mem. Fac. Sci. Kochi. Univ. Ser. A. Math., 17 (1996), 33-42.

[16] A.S. Mashor Abd. El-Monsef M.E. and S.N. Ei-Deeb, On pre continuous and weak precontinuous mapping, Proc. Math., Phys. Soc. Egypt, 53 (1982), 47-53.

[17] O. Njastad, On some classes of nearly open sets, Pacific J. Math., 15 (1965), 961-970. 
[18] N. Nagaveni, Studies on Generalized on Homeomorphisms in Topological Spaces, Ph.D. Thesis, Bharathiar University, Coimbatore, 1999.

[19] O. Ravi and S. Ganesan, g-closed sets in topology, International Journal of Computer Science and Emerging Technologies, 2, No. 3 (2011), 330-337.

[20] S. Sekar and B. Jothilakshmi, On semi generalized star b-closed set in topological spaces, International Journal of Pure and Applied Mathematics, 111, No. 3 (2016).

[21] S. Sekar and B. Jothilakshmi, On semi generalized star b-closed map in topological spaces, International Journal of Pure and Applied Mathematics.

[22] K. Mariappa and S. Sekar, On regular generalized b-continuous map in topological spaces, Kyungpook Mathematical Journal (KMJ), 54 (2014), 477-483.

[23] M.K.R.S. Veerakumar, Between closed sets and g-closed sets, Mem. Fac. Sci. Kochi. Univ., Ser. A: Math., 21 (2000), 1-19. 